\title{
Dynamics of the vapor cavity in the vertical tube of a small diameter after boiling-up in aqueous lithium bromide solutions
}

\author{
Ekaterina Slesareva ${ }^{1,2, *}$, Ruslan Dekhtyar ${ }^{1,2}$, and Valeriy Ovchinnikov ${ }^{1}$ \\ ${ }^{1}$ Kutateladze Institute of Thermophysics, 630090 Novosibirsk, Russia \\ ${ }^{2}$ Novosibirsk State University, 630090 Novosibirsk, Russia
}

\begin{abstract}
Dynamics of a single vapor cavity, moving under gravity in a vertical tube of a small diameter, has been studied. The experiment was carried out in water and aqueous $\mathrm{LiBr}$ solution in a tube with the length of $400 \mathrm{~mm}$ and diameter of $16 \mathrm{~mm}$. Experiments have shown that dynamics of the rising vapor cavity after-the-fact boiling-up of aqueous $\mathrm{LiBr}$ solution in a vertical tube of a small diameter for some initial conditions differs qualitatively from dynamics of a gas bubble rising in a pool. Dynamics of the vapor cavity in aqueous $\mathrm{LiBr}$ solution and in water demonstrates quantitative but not qualitative differences. During bubble rising in a small diameter tube to time dependence for volume and mass of the vapor bubble, the regimes with pulsations were detected.
\end{abstract}

\section{Introduction}

Vapor bubbles are the extremely labile objects. They quickly respond to even slight changes in the ambient pressure or temperature of liquid with which they come into contact. [1-3]. Thus, in the aqueous solution of lithium bromide ( $\mathrm{LiBr}$ ), the velocity of interphase boundary depends on distribution of absorbent concentration. Because of this, experiments with the vapor bubbles rising in the lithium bromide solution are not less significant than experiments with gas bubbles in ordinary liquids. In particular, the importance of influence of various factors on dynamics of the rising vapor bubbles in absorbent solution should be estimated.

When rising, the vapor bubble deforms strongly and moves along an intricate trajectory. For example, Figure 1 shows the video frames of vapor bubble rising in aqueous $\mathrm{LiBr}$ solution of $56 \mathrm{wt} \%$ at temperature $T_{0}=297 \mathrm{~K}$. Vapor at temperature $T_{V 0}=332 \mathrm{~K}$ was fed through the capillary tube in the volume of liquid. Data on volume $V 1$ of the bubble and liquid pressure $P 2$ near the bubble, obtained by processing bubble position and shape in the video frames, are shown in a graph of Figure 1 as a function of time. Knowing the dependence of vapor density on pressure and temperature in the bubble, it is possible to obtain the calculated values of the bubble volume at constant 3 and variable, due to absorption 4 , vapor mass $m$. In the graph, we can see that there is significant discrepancy

\footnotetext{
Corresponding author: styuardessa@yandex.ru
} 
between experimental data and output computation by simplified model, when the bubble approaches the liquid surface in the tube $(t>500 \mathrm{~ms})$. To understand the mechanisms affecting the dynamics of a lifting vapor bubble, a series of experiments in a small-diameter vertical tube with the vapor bubbles produced by boiling-up in water and aqueous $\mathrm{LiBr}$ solution was carried out in this work.

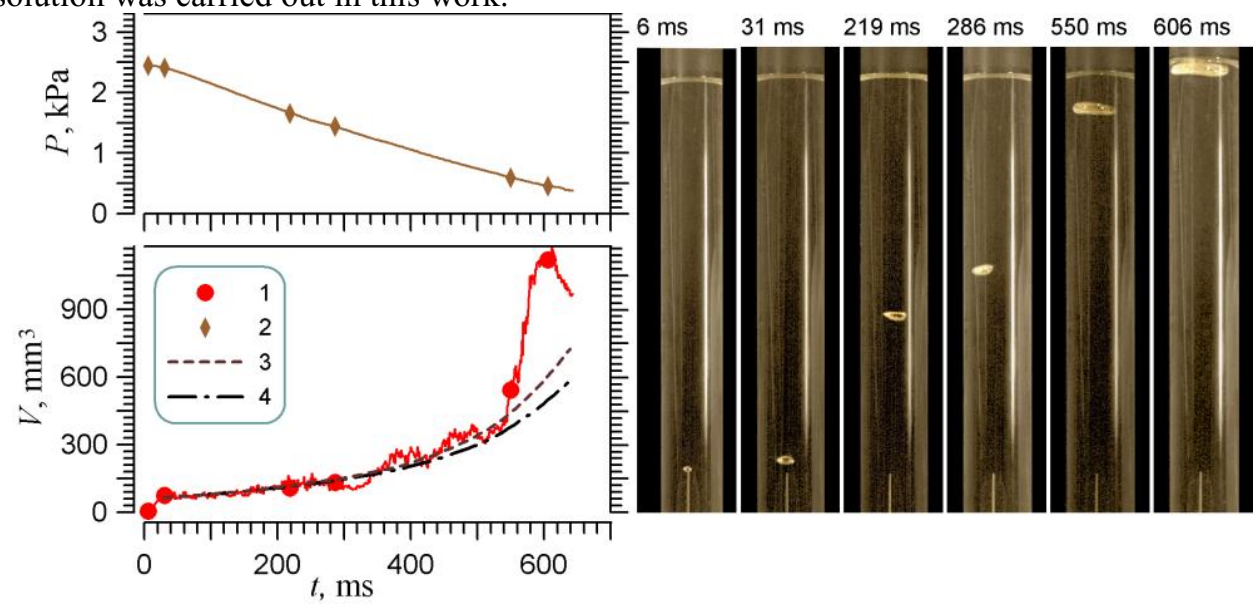

Fig. 1. The video frames of a rising vapor bubble in aqueous $\mathrm{LiBr}$ solution of $56 \mathrm{wt} \%$ at temperature $T_{0}=297 \mathrm{~K}$, vapor temperature $T_{V 0}=332 \mathrm{~K}$. The volume of bubble and liquid pressure near the bubble vs. time: 1 - volume $V, 2$ - pressure $P, 3,4$ - results of calculations of bubble volume ( 3 - with a constant mass of vapor, 4 - with variable mass of vapor reduced because of absorption by $20 \%$ ).

\section{Experimental part}

The schematic diagram of experimental setup is shown in Figure 2. Calibrated glass tube 1 of length $L=400 \mathrm{~mm}$, inner diameter $D=16 \mathrm{~mm}$ was filled with test liquid. Height $H$ of the tube filled with liquid varied from 100 to $200 \mathrm{~mm}$. The setup was vacuumed by vacuum pump 3 through a vacuum trap. The pressure above liquid level was measured by vacuum manometer 2 (precision class 0.25 ). At the height of $10 \mathrm{~mm}$ from the bottom of the tube, electric heater 8 was installed with galvanic isolation. The heater is made of a nichrome tube with the diameter of $6 \mathrm{~mm}$ and wall thickness of $0.5 \mathrm{~mm}$. Tubular heater 9 of $4-\mathrm{mm}$ diameter and length of $16 \mathrm{~mm}$ with copper leads 10 of $3-\mathrm{mm}$ diameter was placed in the nichrome tube. In the central part of the nichrome tube, a chromel-alumel thermocouple was placed. The space between the tube and the heater was filled with finely dispersed electrically insulating paste with a high coefficient of thermal conductivity. The level of heat generation at the heater was set by source of constant current 6 . The voltage drop on the heater varied from 0 to $30 \mathrm{~V}$, electric current varied from 0 to $100 \mathrm{~A}$. To create a hermetic seal between the heater and the working volume, vacuum rubber bushing 7 was used. The temperature of liquid before boiling-up was controlled by infrared imager 5 . For determine the volume of $V_{B}$, surface area $S_{B}$, and position of the vapor bubble by digital video camera 4 at the rate of up to $1000 \mathrm{fps}$ video recording of the vapor bubble and of the liquid level in the tube was performed. The volume of the vapor cavity for different times $t$ was determined from the change in the liquid level height in tube $h_{L}$ and estimated by the formulas taking into account the shape and characteristic dimensions of the vapor bubble.

$$
V_{B}(t)=\pi \cdot \frac{D^{2}}{4} \cdot\left[h_{L}(t)-h_{L}(0)\right]
$$


The pressure in the vapor cavity $P$ was calculated taking into account the height of the liquid column above the bubble and gas pressure in the tube $P_{0}$ in the form:

$$
P(t)=P_{0}+\rho_{L}\left(T_{L}\right) \cdot g \cdot\left[h_{L}(t)-h_{B}(t)\right]
$$

where $h_{B}$ is the height of the bubble top, $\rho_{L}$ is the density of liquid at temperature $T_{L}, \mathrm{~g}$ is acceleration of gravity. The vapor mass in bubble $m$ was calculated using reference data for vapor density $\rho_{V}$ as a function of pressure $P$ and temperature $T$ [4]:

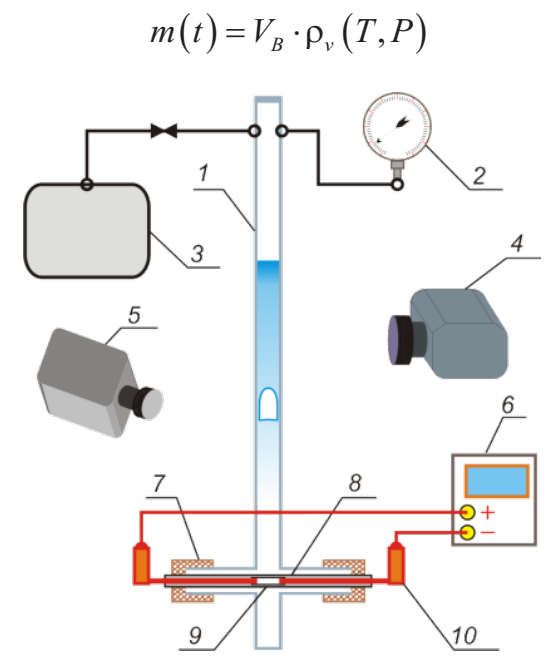

Fig. 2. The scheme of setup: 1 - glass calibrated tube (internal diameter $D=16 \mathrm{~mm}$, length $L=400$ $\mathrm{mm}$ ); 2 - a vacuum manometer; 3 - membrane vacuum pump; 4 - digital video camera; 5 - infrared imager; 6 - DC power supply (voltage $0 \div 30 \mathrm{~V}$, current strength $0 \div 100 \mathrm{~A}$ ); 7 - vacuum rubber bushing; $8,9,10$ - electric heater with galvanic isolation ( 8 - nichrome tube with heat conducting paste and thermocouple temperature sensor, 9 - tubular heater, 10 - copper leads).

\section{Results and discussion}

It was shown that dynamics of the rising vapor cavity after-the-fact boiling-up of aqueous $\mathrm{LiBr}$ solution in a vertical tube of a small diameter for some initial conditions has a qualitative distinction from dynamics of a gas bubble rising in a pool. It should be noted that dynamics of the vapor cavity in aqueous $\mathrm{LiBr}$ solution and water demonstrates quantitative but not qualitative differences. During bubble rising in a small-diameter tube to time dependence of the cavity volume and of the vapor mass, the regimes with pulsation were detected (Figures 3,4). The video frames of the rising vapor bubble in a LiBr solution of $58 \mathrm{wt} \%$ at liquid level above the heater $H_{0}=128 \mathrm{~mm}$, pressure in the volume $P_{0}=518 \mathrm{~Pa}$, solution temperature $T_{0}=297.4 \mathrm{~K}$, and heater temperature before boiling-up $T_{h}=413.4 \mathrm{~K}$ are presented in Figure 3. In these frames, one can see that when floating up, the height of the vapor cavity has nonmonotonic type of relation in time. A graph of varying vapor mass of the vapor cavity in time is shown in Figure 4 for the rising bubble in water and in $\mathrm{LiBr}$ solution. Differences in dynamics of the vapor cavity for water and $\mathrm{LiBr}$ solution can be correlated with parameter $P / P_{0}$ (the ratio of pressure at the heater level to pressure above the liquid surface); this parameter is about 1.5 for water and 4.5 for $\mathrm{LiBr}$ solution (because of a higher density of liquid phase and of a lower density of gas phase). Therefore, differences in duration and amplitude of pulsations in experiments with the rising vapor cavity in tube with water and $\mathrm{LiBr}$ solution are noticeable. For a narrow tube, 
in contrast to pulsations of the vapor bubble in a pool [5], no regimes with shock waves were observed. This is probably due to the fact that the mass of the bubble rising in the tube differs in order of magnitude from the vapor mass in the bubble, which is formed by the action of laser radiation, when shock waves are observed.

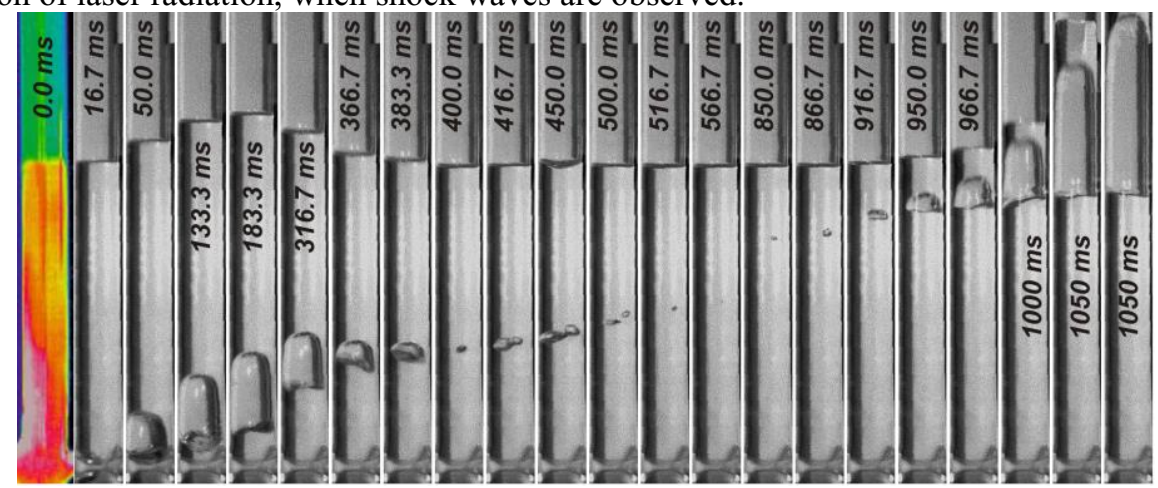

Fig. 3. Video frames after boiling-up of $58 \%$ of aqueous $\mathrm{LiBr}$ solution in tube $D=16 \mathrm{~mm}$, $H_{0}=128 \mathrm{~mm}, P_{0}=518 \mathrm{~Pa}, T_{0}=297.4 \mathrm{~K}, T_{h}=413.4 \mathrm{~K}$.

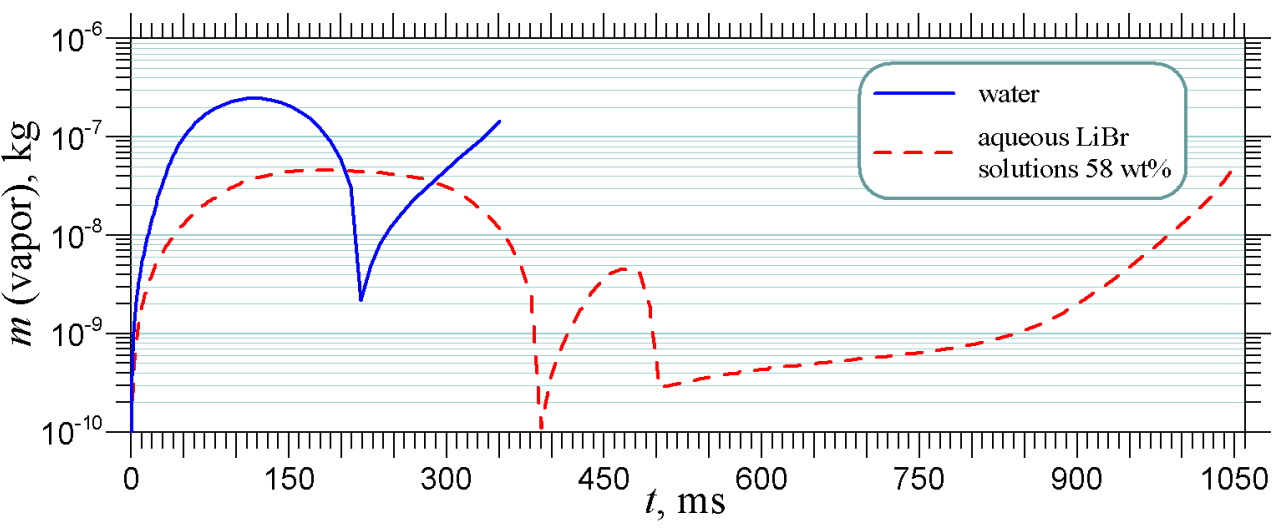

Fig. 4. Variation vapor mass in the vapor cavern in time for the rising bubble in water $\left(H_{0}=98 \mathrm{~mm}\right.$, $\left.P_{0}=2730 \mathrm{~Pa}, T_{0}=295.6 \mathrm{~K}, T_{h}=361.1 \mathrm{~K}\right)$ and in aqueous $\mathrm{LiBr}$ solution $58 \mathrm{wt} \%\left(H_{0}=128 \mathrm{~mm}\right.$, $\left.P_{0}=518 \mathrm{~Pa}, T_{0}=297.4 \mathrm{~K}, T_{h}=413.4 \mathrm{~K}\right)$.

The acknowledgements: the study was funded by FASO of Russia.

\section{References}

1. A. Prosperetti, Ann. Rev. Fluid Mech. 49, 221 (2017)

2. V .E. Nakoryakov, S. Y. Misyura, J. Engng. Thermophys. 25, 24 (2016)

3. St. Kiirner, L. Friedel, J. Loss, Prev. Process Ind. 10, 221 (1997)

4. H. Lover, Thermodynamischen und physikalische Eigenschaften der wässrigen Lithiumbromid Lösung (Dissertation, Karlsruhe, 1960)

5. I. Akhatov, O. Lindau, A. Topolnikov, R. Mettin, N. Vakhitova, W. Lauterborn, Phys. Fluids. 13, 2805 (2001) 